\section{Obama 2016 budget calls for Precision Medicine}

In his 2016 budget proposal, President Barack Obama seeks $\$ 31.3$ billion for the National Institutes of Health ( $\mathrm{NIH})$, an increase of $\$ 1$ billion over 2015 levels. The proposal also announces a Precision Medicine initiative, calling for $\$ 216$ million in 2016 for efforts at $\mathrm{NIH}$, the Food and Drug Administration (FDA) and the Office of the National Coordinator for Health Information Technology. This initiative is intended "to develop prevention, diagnostic, and treatment approaches tailored to individual patients," officials note. Elsewhere, the budget proposes $\$ 1.2$ billion for federal programs to combat and prevent antibiotic resistance, with those resources shared among NIH, FDA, the Centers for Disease Control and Prevention and the Biomedical Advanced Research and Development Authority, as well as the Departments of Veterans Affairs and Defense. Amid these proposals to support biomedical research, Washington, DC-based Biotechnology Industry Organization says that parts of the proposed 2016 budget that call for reducing the period of regulatory data protection for innovative biologics as well as several proposed changes to Medicare "will directly undermine other initiatives and activities contained within the President's budget and which we find critically important."

"We looked back at the slides we
used in the IPO [in 2000]—-they are
embarrassing." George A. Scangos, former
CEO of San Francisco-based Exelixis,
commenting on the difference between this
biotech boom and the one in 2000. (The
New York Times, 18 January 2015)
"I don't think in any way that we have licked
the problem. Since we don't know how the
machine works, we don't know what to do
when it breaks." Roger M. Perlmutter, head
of R\&D at Merck, speaking of the difficulties
faced by drug developers. (The New York
Times, 18 January 2015 )
"I have my seatbelt and crash helmet on."
Chief executive of a biotech company,
speaking anonymously to a reporter at the
JP Morgan Healthcare Conference, held in
January in San Francisco. This executive
company's stock price has tripled in price
since last spring. (The New York Times,
18 January 2015)
"There is no selectivity in abundance. With
enough fertilizer, everything will grow-
weeds and all." Kevin Johnson, partner at
Index Ventures, commenting on the biotech
bubble. (Forbes, 21 January 2015)
"This is an experiment we are poised to do.
It is not a giant 'put a man on the moon'
type project. It is a very tractable question."
Charles Sawyers, from the Memorial
Sloan Kettering Cancer Center in New
York, commenting on the White House's
"precision medicine" plan. (The New
York Times, 2 February 2015)

to harmonize clinical trial data collection and analysis."

Independent of Roche, Foundation Medicine has its sights set on developing a universal cancer diagnostic-a goal shared by several companies including sequencing leaders Illumina, in San Diego, and Thermo Fisher's Life Technologies, in Carlsbad, California. Each sequencing outfit set up consortia last year with leading pharma companies (Nat. Biotechnol. 32, 1073, 2014) to develop diagnostic tests that would be used broadly to direct therapy choices, in many cases as a set of companion diagnostics linked to prescribing particular drugs.

A companion diagnostic developed using the Foundation Medicine platform would be considered a laboratory-developed test (LDT). The biotech is currently partnering with Clovis Oncology, in Boulder, Colorado, on such a companion diagnostic using its commercial FoundationOne platform to detect a subset of markers relevant to the Clovis phase 2 ovarian cancer drug candidate rucaparib, an inhibitor of poly (ADP-ribose) polymerase (PARP). To that end, Foundation Medicine is building a dedicated laboratory that Clovis will use for testing.

Recently, Myriad Genetics, in Salt Lake City, Utah, secured approval of a companion diagnostic to be used with London-based AstraZeneca's first-in-class PARP inhibitor, Lynparza (olaparib) - the first time the US Food and Drug Administration (FDA) approved an LDT test and a drug simultaneously. Myriad's US-approved companion diagnostic, BRACAnalysis CDx, looks only at germline mutations. Foundation Medicine's test would include identification of somatic as well as germline mutations. (Myriad has, however, opened a laboratory in Europe that performs germline and somatic testing.)

With companion diagnostics' growing importance in the development of precision medicines, pharma's interest in gaining stakes in diagnostics companies makes sense. "Pharma companies are deep-pocketed partners with a great deal of FDA experience, and the agency eventually wants to regulate
LDTs," says Marc Samuels, president and CEO of ADVI, a Washington, DC-based healthcare advisory services firm. "Greater ownership of a diagnostics firm allows a pharma to better manage and control the fate of its pipeline in an era where biomarkers are the key to faster approvals with smaller clinical trials."

Although the leverage Roche is providing Foundation Medicine is what diagnostic companies seek, a different model is emerging in the UK. $14 \mathrm{M}$ Genomics is a Cambridge-based spinout of the Wellcome Trust Sanger Institute, which is establishing partnerships with patient organizations to undertake cancer gene studies on patients and link clinical diagnosis, treatment and outcomes data. These partnerships will provide well-recorded clinical data from tens of thousands of patients to follow what happens in their treatment life. 14M Genomics will undertake genomic studies on patient samples and apply its bioinformatics tools to interpret the complex signatures underpinning the biology of disease. "That's the piece we really have not seen anybody else do at scale," says executive chairman Andrew Sandham. In February, $14 \mathrm{M}$ Genomics signed a collaboration with the Haematological Malignancy Research Network to map patterns of genomic variation in patients with hematological cancers and link them with clinical outcomes. 14M Genomics expects to start to roll out services to oncologists in two years. "We are not under pressure to make numbers, so we can afford to invest deeply in building those reference data sets and having them available," he says, adding that in Europe, unlike the LDT model in the US, clinical sequencing will likely be distributed across many laboratories.

Foundation Medicine envisages continuing down the LDT path "because that's what's well defined for us," says Kafka. However, diagnostic kits are also part of the planned collaborations with Roche. Foundation Medicine is "absolutely interested" in pursuing an in vitro diagnostic regulatory path as well, Kafka says.

Mark Ratner Boca Raton, Florida 